\title{
State of the Art of Dual Therapy in 2015
}

Article in AIDS reviews · July 2015

Impact Factor: 3.79

READS

85

10 authors, including:

\section{Silvia Nozza}

Università Vita-Salute San Raffaele

93 PUBLICATIONS $\mathbf{1 , 5 5 5}$ CITATIONS

SEE PROFILE

Annalisa Saracino

Università degli studi di Foggia

93 PUBLICATIONS 1,029 CITATIONS

SEE PROFILE

\section{Valentina Svicher}

University of Rome Tor Vergata

152 PUBLICATIONS 1,198 CITATIONS

SEE PROFILE

Antonio Di Biagio

Azienda Ospedaliera Universitaria San Mart...

198 PUBLICATIONS 1,214 CITATIONS

SEE PROFILE 


\title{
State of the Art of Dual Therapy in 2015
}

\author{
Silvia Nozza ${ }^{1}$, Valentina Svicher ${ }^{2}$, Annalisa Saracino ${ }^{3}$, Gabriella d'Ettorre ${ }^{4}$, Andrea De Luca ${ }^{5}$, Franco Maggiolo ${ }^{6}$, \\ Stefano Bonora 7 , Antonio di Biagio ${ }^{8}$, Stefano Rusconi ${ }^{9}$ and Cristina Mussinit ${ }^{10}$ \\ ${ }^{1}$ Clinic of Infectious Diseases, San Raffaele Scientific Institute, Milan, Italy; ${ }^{2}$ University of Tor Vergata, Rome, Italy; ${ }^{3}$ Clinic of Infectious Diseases, \\ University of Bari, Bari, Italy; ${ }^{4}$ Clinic of Infectious Diseases, Policlinico Umberto I, Rome, Italy; ${ }^{5}$ University Hospital of Siena, Siena, Italy; ${ }^{6} \mathrm{Hospital}$ \\ Giovanni XXIII, Bergamo, Italy; ${ }^{7}$ Clinic of Infectious Diseases, University of Turin, Turin, Italy; ${ }^{8}$ Clinic of Infectious Diseases, Genova, Italy; ${ }^{9}$ Clinic of \\ Infectious Diseases, University of Milan, Milan, Italy; ${ }^{10}$ Clinic of Infectious Diseases, University of Modena and Reggio Emilia, Modena, Italy
}

\begin{abstract}
Dual therapy refers to combinations of two antiretroviral drugs applied in different clinical settings; they are considered and studied due to possibly reduced drug toxicities. In antiretroviral-naive patients, dual combinations have lower virologic efficacy than standard therapy; the sole efficacious regimen is lamivudine plus lopinavirl ritonavir. Due to a higher possibility of virologic failure, these regimens are generally not allowed in this clinical setting. In antiretroviral-experienced patients, dual regimens are examined in studies with a small sample size, centered on clinical practice, and should be ritonavir-boosted protease inhibitor-based. These combinations have a good virological efficacy; combinations with the integrase inhibitor raltegravir have small sample size and demonstrated efficacy only with etravirine. Virological aspects involving dual therapy should always consider genetic barriers, particularly in simplification strategies, and ritonavir-boosted protease inhibitors are mandatory. As far as immunological aspects are concerned, nucleoside reverse transcriptase inhibitorsparing regimens have some encouraging data, probably due to the bone marrow toxicity of this class. Combinations with maraviroc were effective in reducing inflammation, but data about immunological recovery are conflicting. The choice of regimen should focus on specific class toxicity since dual regimens are studied in particular for improving safety and tolerability. This review will analyze different dual regimens in the clinical setting, with a peculiar focus on ameliorating toxicities and improving quality of life. (AIDS Rev. 2015;17:127-34) Corresponding author: Cristina Mussini, crimuss@unimore.it
\end{abstract}

\section{Key words}

Dual therapy. HAART. Simplification. Toxicity,

\section{Introduction}

Antiretroviral strategies with fewer drug regimens are currently being considered and studied in clinical practice. Dual therapy refers to combinations of two

Correspondence to:

Cristina Mussini

Clinic of Infectious Diseases

Policlinico

Via del pozzo, 71

41100 Modena, Italy

E-mail: crimuss@unimore.it antiretroviral (ARV) drugs applied in different clinical settings; the virologic efficacy of these combinations are potentially less than triple standard regimens of combined ARV therapy (HAART). The main benefits are reduced drug-related toxicities.

We will review the virologic efficacy of these combinations in different clinical settings and analyze toxicities and drug-drug interactions.

\section{Dual therapy in antiretroviral-naive patients}

The current recommended ARV treatment for naive $\mathrm{HIV}$-1-infected patients is a combination of two nucleoside/nucleotide reverse transcriptase inhibitors (NRTI) 
Table 1. Dual therapy in antiretroviral naive patients; virologic efficacy is percentage of patients with HIV/RNA $<50$ copies/ml

\begin{tabular}{|c|c|c|c|}
\hline Study & Standard group & Dual group & $\begin{array}{l}\text { Virological efficacy at week } 48 \\
\text { or discontinuation* }\end{array}$ \\
\hline PROGRESS ${ }^{3}$ & TDF/FTC + LPV/r & $R A L+L P V / r$ & $\begin{array}{l}\text { TDF/FTC + LPV/r: } 84.2 \% \\
\text { RAL + LPV/r: } 83.2 \%\end{array}$ \\
\hline VEMAN ${ }^{4}$ & TDF/FTC + LPV/r & MVC 150 mg QD + LPV/r & $\begin{array}{l}\text { TDF/FTC + LPV/r: } 100 \% \\
\text { MVC } 150 \text { mg QD + LPV/r: 100\% }\end{array}$ \\
\hline MODERN ${ }^{9}$ & TDF/FTC + DRV/r (800/100 mg) & MVC $150 \mathrm{mg}$ QD + DRV/r & $\begin{array}{l}\text { TDF/FTC + DRV/r (800/100 mg): 83\%* } \\
\text { MVC } 150 \mathrm{mg} \text { QD + DRV/r: 72\%* }\end{array}$ \\
\hline SPARTAN $^{6}$ & $T D F / F T C+A T V / r$ & $R A L+A T V / r$ & $\begin{array}{l}\text { TDF/FTC + ATV/r: } 63.3^{*} \\
\text { RAL + ATV/r: } 73.6 \%^{*}\end{array}$ \\
\hline A4001078 & $T D F / F T C+A T V / r$ & MVC $150 M G Q D+A T V / r$ & $\begin{array}{l}\text { TDF/FTC + ATV/r: } 83.6 \% \\
\text { MVC + ATV/r: } 74.6 \%\end{array}$ \\
\hline NEAT-0017 & TDF/FTC + DRV/r (800/100 mg) & $R A L+D R V / r$ & $\begin{array}{l}\text { TDF/FTC + DRV/r (800/100 mg): } 94 \% \\
\text { RAL + DRV/r: } 89 \%\end{array}$ \\
\hline GARDEL $^{10}$ & 2 NRTIs + LPV/r & $3 T C+L P V / r$ & $\begin{array}{l}2 \mathrm{NRTIS}+\mathrm{LPV} / \mathrm{r}: 83.7 \% \\
3 \mathrm{TC}+\mathrm{LPV} / \mathrm{r}: 88.3 \%\end{array}$ \\
\hline
\end{tabular}

and a third agent from another class: integrase strand transfer inhibitor (INSTI) or ritonavir-boosted protease inhibitor $(\mathrm{Pl} / \mathrm{r})^{1}$. Tenofovir/emtricitabine (TDF/FTC) in combination with INSTI (raltegravir, dolutegravir, elvitegravir/cobicistat) or $\mathrm{Pl} / \mathrm{r}$ (darunavir/ritonavir), and abacavir/lamivudine (ABC/3TC) in combination with dolutegravir (DTG) are the preferred regimens.

The NRTI-sparing regimens are an alternative in naive patients, but generally not recommended, and should be considered in the presence of comorbidities contraindicating the use of NRTIs or in the presence of resistance to NRTIs. Actual data do not recommend this strategy to prevent toxicity.

Lopinavir/ritonavir (LPV/r), atazanavir/ritonavir (ATV/r), and darunavir/ritonavir (DRV/r) have been considered in clinical trials combined with raltegravir (RAL) or maraviroc (MVC) $150 \mathrm{mg}$ once daily (NRTI-sparing regimens) $)^{2-5}$. Table 1 summarizes the virological response at week 48 of these regimens. This response is generally inferior in dual regimens, with the exception of ATV/r plus RAL and LPV/r plus MVC.

These studies were proof-of-concept and enrolled a limited number of subjects. Two trials with DRV/r $800 / 100 \mathrm{mg}$ once daily have been designed with the sample size calculation to demonstrate non-inferiority of the NRTI-sparing regimen. In a single-arm study (ACTG A5262), DRV/r was combined with RAL. In 112 treatment-naive subjects (median HIV RNA level $4.9 \log _{10}$ copies $/ \mathrm{ml}[44 \%>100,000$ copies $/ \mathrm{ml}]$ and median CD4 count 271 cells/ $\mu$ l) a high rate of virologic failure was confirmed at week 24 and week 48: only 79 and $71 \%$, respectively, had HIV/RNA levels < 50 copies $/ \mathrm{ml}$; 28 subjects had confirmed virologic failure. Integrase resistance was found in five of 25 subjects in whom resistance testing was done; virologic failure was associated with higher baseline HIV/RNA (odds ratio for RNA > 100,000 copies/ml: 3.76) and with lower CD4 counts ${ }^{6}$. In the randomized NEAT-001 trial $^{7}$, the superiority of triple ARV therapy (TDF/FTC plus DRV/r) versus dual therapy with RAL plus DRV/r was evidenced at week $48: 88.5$ and $83.7 \%$ of subjects had HIV/RNA < 50 copies/ml in patients with baseline HIV/RNA > 100,000 copies/ml, respectively.

A different study with DRV/r plus MVC $150 \mathrm{mg}$ once daily (A4001095, MODERN trial) was designed. The study was terminated in October 2013 following a preliminary interim analysis of week 48 primary efficacy data by the study's external independent Data Monitoring Committee. The Committee assessed the data as demonstrating significant differences between the treatment arms in virologic responses (72\% in the maraviroc group, $83 \%$ in the standard regimen) and failures. They recommended, and the Sponsor concurred, that the study should be terminated because of the inferior efficacy of the MVC arm as compared to the comparator arm (emtricitabine/tenofovir) ${ }^{8}$.

In selected patients, dual therapy with $\mathrm{PI} / \mathrm{r}$ plus 3TC could be an option. In ARV-naive patients, a randomized 
open-label study (GARDEL) demonstrated that LPV/r plus 3TC was non-inferior to triple therapy after 48 weeks of treatment, regardless of baseline viral load; at week 48, $88.3 \%$ of subjects who received dual therapy and $83.7 \%$ of those treated with triple standard therapy were responders ${ }^{9}$.

\section{Dual therapy in antiretroviral-experienced patients}

Few trials have been performed in this clinical setting, although simplification from a more traditional HAART to a NRTI-sparing regimen appears to be a very fascinating hypothesis.

Combinations of $\mathrm{Pl} / \mathrm{r}$ and nucleoside reverse transcriptase inhibitors (NNRTI) have been tested in several situations. The pilot randomized study NEKA evaluated the safety and efficacy of a combination of LPV/r and nevirapine (NEV) in 31 HIV-1-infected subjects with stable virologic suppression ${ }^{10}$. This association was shown to be effective after a follow-up of 48 weeks. No subjects discontinued therapy because of adverse events. The MULTINEKA study analyzed 67 HIV-1-infected subjects who were switched to a NRTI-sparing regimen including NEV and LPV/ $/ \mathrm{r}^{11}$. This study demonstrated an equivalent efficacy of the dual therapy when compared to the traditional triple HAART.

Switching to DRV/r plus etravirine (ETR) was evaluated in 20 patients $^{12}$ : 65\% (13/20) switched from dual $\mathrm{Pl} / \mathrm{r}$ regimens, eight combined with efavirenz (EFV) or NEV, and 20\% (4/20) switched from conventional HAART. Patients had a median exposure to nine ARV drugs prior to switch (range 3-14), with 90\% (18/20) having previous NNRTI exposure; at switch, 60\% (12/20) had no previous resistance, 25\% (5/20) NRTI mutations only, and 15\% (3/20) had NNRTI mutations. At week 24, all patients maintained undetectable viral load.

Other PI/r-based switch strategies have been tested in different settings. The KITE study analyzed the therapeutic switch from standard HAART to LPV/r plus RAL and evidenced a comparable virologic efficacy at week 48 ( 88 vs. $92 \%)^{13}$. The ATLAS study was a singlearm study, which analyzed virologically suppressed HIV-1-infected subjects who were treated with TDF/ FTC plus ATV/r after switching them to a dual regimen containing 3TC plus ATV/ $\mathrm{r}^{14}$. The ATLAS study was conducted in $40 \mathrm{HIV}$-1-infected subjects. After 48 weeks, 4/40 (10\%) regimen discontinuations occurred: one death (brain hemorrhage), one study withdrawal (inadequate ATV plasma levels), one re-induction with two NRTIs due to pregnancy, and one virologic failure without development of resistance. The positive results of ATLAS supported the design of a multicenter randomized clinical trial, which is currently ongoing. In a similar Spanish study $(S A L T)^{15}$, dual therapy with ATV/r plus 3TC appeared to be as safe and effective in the short term as switching therapy in virologically stable patients requiring a change in treatment owing to simplification, intolerance, or toxicity. If these encouraging data are confirmed, this strategy could be a good alternative to monotherapy and would avoid TDF toxicity without the disadvantages of monotherapy. An observational, uncontrolled, real-life study using the oncedaily regimen DRV/r 800/100 mg plus MVC $150 \mathrm{mg}$ enrolled 60 HIV-1-positive subjects coming from traditional triple therapy and switched to dual therapy ${ }^{16}$; 44 (73\%) patients reached HIV/RNA < 50 copies/ml at week 48 . The MITOX study ${ }^{17}$ randomized 40 out of 80 patients who had received two NRTI plus PI/r to continue their current regimen or switch to a $\mathrm{Pl} / \mathrm{r}$ plus MVC $150 \mathrm{mg}$ once daily. Six out of 40 patients (15\%) receiving MVC plus PI/r failed; four of them received DRV/r plus MVC, and only one out the six failing patients exhibited a switch in tropism toward a X4-tropic virus.

In the other strategies, MVC plus RAL and RAL plus ETR were studied. The ROCnRAL trial ${ }^{18}$ is a single-arm study that switched 44 patients from a suppressive HAART to MVC 300 mg twice daily plus RAL 400 mg twice daily with $\mathrm{R} 5$ tropic virus and undetectable viral load from 5.2 years (IQR: 4.4-7.9), nadir CD4 210 cells $/ \mathrm{mm}^{3}$ (IQR: 150-276), HAART duration 15 years (IQR: 15-19); seven (16\%) patients failed MVC/RAL therapy: five with virological failure and two discontinued treatment due to adverse events. The high rate of virologic failure of this dual combination was confirmed by an Italian study: 9/26 (35\%) multi-experienced patients failed simplification therapy with MVC plus RAL at week $24^{19}$. The MVC plus RAL was effective in 10 naive patients treated for 24 weeks with an induction therapy (TDF/FTC plus RAL plus MVC) and then switched to dual therapy: after 48 weeks, undetectable viral load was maintained in all patients ${ }^{20}$. These different results are not comparable due to patient characteristics (multi-experienced versus naive).

Eighteen patients with six years of viral suppression were switched to RAL plus ETR regimens, with undetectable viral load during 12 months of follow-up ${ }^{21}$; in another recent study including 25 patients, this switch maintained virologic suppression at week 48 in $84 \%$ of patients ${ }^{22}$.

Dual therapies in experienced patients are summarized in table 2. 
Table 2. Dual therapy in antiretroviral experienced patients; virologic efficacy is percentage of patients with HIV/RNA $<50$ copies/ml

\begin{tabular}{|c|c|c|c|}
\hline Study & Standard group & Dual group & $\begin{array}{l}\text { Virological efficacy at week } 48 \\
\text { or discontinuation* }\end{array}$ \\
\hline MULTINEKA ${ }^{12}$ & $2 \mathrm{NRTIS}+\mathrm{LPV} / \mathrm{r}$ & $N E V+L P V / r$ & $\begin{array}{l}2 \text { NRTIs + LPV/r: } 60.6 \% \\
\text { NEV + LPV/r: } 81.8 \%\end{array}$ \\
\hline $\mathrm{KITE}^{14}$ & $2 \mathrm{NRTIS}+\mathrm{LPV} / \mathrm{r}$ & $R A L+L P V / r$ & $\begin{array}{l}2 \text { NRTIs + LPV/r: } 88 \% \\
\text { RAL + LPV/r: } 92 \%\end{array}$ \\
\hline ATLAS $^{15}$ & NA & $3 T C+A T V / r$ & $3 T C+A T V / r: 90 \%$ \\
\hline MITOX ${ }^{18}$ & 2 NRTIs + Pl/r & MVC $150 \mathrm{mg} \mathrm{QD} \mathrm{+} \mathrm{PI/r}$ & $\begin{array}{l}2 \text { NRTIs + Pl/r: } 100 \% \\
\text { MVC } 150 \mathrm{mg} \text { QD + Pl/r: } 85 \%\end{array}$ \\
\hline ROCnRAL $^{19}$ & NA & $M V C+R A L$ & MVC + RAL: $86 \% *$ \\
\hline $\mathrm{RAL}+\mathrm{ETR}^{7}$ & NA & $R A L+E T R$ & RAL + ETR: $84 \%$ \\
\hline
\end{tabular}

3TC: lamivudine; /r: ritonavir boosted; ATV: atazanavir; ETR: etravirine; FTC: emtricitabine; LPV: lopinavir; MVC: maraviroc; NEV: nevirapine; NRTI: nucleoside/nucleotide reverse transcriptase inhibitor; PI: protease inhibitor; QD: once daily; RAL: raltegravir.

\section{Virologic aspects}

So far, the identification of virologic factors that can help identify patients as candidates for dual therapy represents a challenge in the field of HIV-1 clinical research.

In patients starting a first-line $\mathrm{Pl} / \mathrm{r}$-based dual therapy, the level of plasma HIV-1 RNA at baseline represents an important parameter. In particular, a baseline viral load >100,000 copies/ml strongly correlates with an increased risk of virologic failure in patients receiving DRV/r plus RAL ${ }^{7}$. In the setting of a higher baseline viral load, the selective pressure imposed by two drugs may take a prolonged time to achieve virologic suppression, thus predisposing to the generation of drug resistance mutations, and in turn to virologic rebound.

The duration of viral suppression ${ }^{23}$ and/or the occurrence of residual viremia ${ }^{24}$ might also influence the probability of maintaining the virologic success in aviremic patients switching from a triple ARV regimen to $\mathrm{Pl} / \mathrm{r}$-based dual therapy. The role of these factors has not been adequately investigated in dual therapy strategies so far, but some suggestions could be derived from studies on $\mathrm{Pl} / \mathrm{r}$ monotherapy ${ }^{25,26}$. Moreover, several studies have highlighted a strict correlation between the level of baseline viral load and the burden of the cellular reservoir quantified by cellular HIV-1 DNA ${ }^{27}$. In this setting, dual therapy may account for a cryptic ongoing viral replication, which could favor the emergence of drug resistance. This is consistent with a recent study showing that long-term virologic success on dual therapy with two NRTIs correlates with a low level of HIV-1 DNA (median 2.5 log copies $/ 10^{6}$ peripheral blood mononuclear cells ${ }^{28}$. In this study, another factor associated with the maintenance of virologic suppression in patients receiving a two-NRTI-based therapy is early HAART initiation. Since early treatment has been associated with a reduced burden of HIV-1 cellular reservoir, these results highlight the need to investigate a potential threshold of HIV-1 DNA so that dual therapy can be safely administered as first-line therapy.

Another issue that deserves further investigation is the genetic barrier of the PI/r companion drug. In the ACTG A5262 study ${ }^{7}$, virologic failures were associated with the emergence of mutations associated with resistance to RAL. The SPARTAN study has been prematurely terminated due to the frequent emergence of RAL resistance mutations in patients experiencing virologic failure ${ }^{5}$. Similarly, dual therapy with LPV/r plus EFV was associated with high rates of NNRTI resistance during virologic failure ${ }^{29}$. Therefore, the genotypic sensitivity score as well as genotypic resistance testing on proviral DNA should be included in the evaluation of potential candidates for a dual-ARV regimen. Further studies addressing the issue of the genetic barrier of the companion drug are urgently needed for safe administration of dual therapy in drug-naive patients. Moreover, when choosing a dual regimen including MVC, a proper assessment of coreceptor tropism is mandatory since the sensitivity of the tropism test (phenotypic or genotypic) to detect minority nonR5 variants is crucial ${ }^{30,31}$.

\section{Immunological aspects}

An adequate immunological response during HAART is defined as an increase in CD4+ $\mathrm{T}$-cells counts in the 
range of 50-150 per year, generally with an accelerated response in the first three months of treatment. However, the reconstitution of CD4 ${ }^{+} \mathrm{T}$-cells is variable among patients, depending on different factors such as nadir of $\mathrm{CD}^{+}$, aging, and comorbidities. Furthermore, the immune response during HAART includes not only the increase of $\mathrm{CD} 4^{+}$T-cells, but also the status of immune activation ${ }^{32}$.

Recent studies on pathogenesis report a direct correlation between persistent immune activation/inflammation and higher levels of microbial translocation, with a poor recovery of $\mathrm{CD}^{+}{ }^{+} \mathrm{T}$-cells in individuals suppressed with HAART for a long time ${ }^{33-36}$.

Moreover, recently published guidelines (US Department of Health and Human Services, European Aids Clinical Society, GeSIDA, Italian Ministry of Health) suggest including also the measurement of soluble markers of the immune activation such as lipopolysaccharide, CD16, and others for immunological monitoring of patients. In this context, some of the studies of dual therapy based on $\mathrm{Pl} / \mathrm{r}$ in combination with one NRTI or RAL or MVC or NNRTI analyzed immunological aspects: both $\mathrm{CD}^{+}{ }^{+} \mathrm{T}$-cell response and the markers of immune activation. Soluble CD14 (sCD14) was associated with mortality ${ }^{37}$ and lipopolysaccharide with clinical progression of HIV infection, independently from $\mathrm{CD}^{+}$and HIV/RNA ${ }^{38}$.

Both MVC and RAL have a distinctive role in decreasing T-cell activation. While dual therapy including MVC has been studied in patients with incomplete recovery of $\mathrm{CD} 4^{+} \mathrm{T}$-cells or naive for HAART, regimens including RAL have been explored as first-line therapy and in simplification studies. The immunomodulatory effect of MVC is related to the function of the coreceptor CCR5. The CCR5 has an important role in the pathogenesis of HIV infection and in the control of proinflammatory effects: the dysregulation of CCR5mediated lymphocyte trafficking has been associated to several inflammatory conditions such as rheumatoid arthritis and organ transplants. In this context, a different tissue distribution of $\mathrm{CCR} 5^{+}$and $\mathrm{CD} 25^{+} \mathrm{T}$-cells was observed, with a possible decrease in the levels of immune activation after administration of antagonistic anti-CCR5 monoclonal antibody to rhesus macaques ${ }^{39}$.

However, the results in vivo on the effect of MVC to modulate the immune activation are discordant. Hunt, et al. compared the effect of MVC intensification in patients under HAART with CD4+ ${ }^{+}$-cell counts $<350$ cells $/ \mathrm{mmc}$ and plasma levels < 48 copies $/ \mathrm{ml}$ on peripheral immune activation and on gut-associated lymphoid tissue versus placebo after 24 weeks of treatment. The authors observed a twofold increase in T-cell activation in rectal tissue and a lower but statistically significant increase in peripheral blood after an intensification regimen ${ }^{40}$. On the contrary, the ACTG study (A5256) conducted in patients with incomplete recovery of $\mathrm{CD} 4^{+} \mathrm{T}$-cells showed an apparent reduction of T-cell activation, with a decline in CD38 expression and increased HLA-DR, with a partial reversion after the interruption of $\mathrm{MVC}^{41}$.

The intensification with MVC in immunological nonresponders in a multicentric, randomized, open label, phase IV superiority trial did not demonstrate a significant advantage in reconstituting the $\mathrm{CD}^{+}{ }^{+} \mathrm{T}$-cell pool in terms of increases in $\mathrm{CD}^{+}$cells and parameters of T-cell homeostasis and activation. Patients receiving MVC experienced a significant rise in circulating interleukin 7 by week $48(p=0.01)$ and a trend in temporary reduction in activated HLA-DR+CD38+CD4+ by week $12(p=0.06)$ that was not maintained at week $48^{42}$.

The ability of HAART to decrease viral load drives the decline of immune activation and influences the survival of patients. The important effect of RAL on the decay of viral load reflects its ability to control the immunological response to dual therapy including RAL and $\mathrm{Pl} / \mathrm{r}^{43}$, probably due to the direct correlation between the reduction of viral load and of the markers of immune activation ${ }^{7}$.

\section{Safety aspects}

Increasing attention has been focused on the longterm adverse effects of HAART, including fat distribution changes observed in lipodystrophy syndrome, the increased risk of cardiovascular disease, and the onset of bone and kidney diseases ${ }^{44}$. The NRTI- and $\mathrm{Pl}$-sparing regimens are attractive options to avoid the toxicity associated with NRTIs and high doses of ritonavir.

\section{Dual protease inhibitor and nucleoside reverse transcriptase inhibitor-sparing regimens}

In this scenario there are few reported data on kidney and metabolic aspects. The reasons for switching from PIs were mostly toxicity related: metabolic disorder and/or lipodystrophy. In two different studies of switching to dual therapy based on RAL plus entecavir (ETV), the authors registered a decrease in median cholesterol, triglyceride levels, and glucose levels ${ }^{21,22}$. The French study ROCnRAL was based on the switch 
to dual therapy with RAL plus MVC in patients with lipodystrophy: lipid profiles improved with a decrease from baseline values in total cholesterol, but two patients discontinued treatment due to severe adverse events (HBV rebound in a patient $\mathrm{HBCAb}^{+}$and $\mathrm{HBsAg}^{-}$, one hypersensitivity syndrome $)^{18}$. Due to the limited number of patients and the observational and nonrandomized nature of the study, all these data should be carefully considered. Scarce data are available regarding kidney function.

\section{Dual nucleoside reverse transcriptase inhibitor-sparing regimens}

Although today's NRTIs are safer than first-generation agents in this class, they still carry some risks of long-term toxicity. No international guidelines recommend NRTI-sparing regimens for first-line or later regimens, but the availability of newer ARVs is making such combinations more popular in practice. An increase in lipid values was observed in patients treated with NRTI-sparing regimens ${ }^{45}$. Furthermore, patients on a combination of PI plus NNRTI were more likely to have an atherogenic lipid profile than patients on Pls only ${ }^{46}$.

In most recent studies on NRTI-sparing regimens conducted in observational cohorts, the authors observed a decrease in triglycerides. Cholesterol and creatinine, by contrast, did not improve and there were no significant differences in hepatic metabolism. It is important to highlight that patients received NRTI-sparing regimens without ritonavir in these two studies ${ }^{47}$.

In the KITE study, the switch to RAL plus LPV/r was associated with increases in fasting plasma total cholesterol, triglycerides, and LDL-cholesterol levels at 24 weeks, but only the increase in triglyceride levels was statistically significant ${ }^{18}$.

In the PROGRESS study, LPV/r plus RAL was not associated with a decline in renal function at week 96 . In the NRTI-sparing arm, the investigators observed hyperlipidemia and diarrhea, which is consistent with the established profile for LPV/r. There were minimal changes from baseline in TC:HDL ratios, LDL:HDL ratios, and 10-year Framingham cardiovascular risk scores based on LDL and total cholesterol levels in both treatment groups.

On the contrary, in the SPARTAN trial, the overall profile of ATV plus RAL did not appear optimal for further clinical development, given higher rates of resistance to ARVs and hyperbilirubinemia with twicedaily ATV5.
In lipoatrophic patients, small studies evaluated the benefit of a switch to a PI-containing/NRTI-sparing regimen compared with maintenance of a NRTI-containing regimen, and showed that the combination LPV/r plus EFV was associated with a significant improvement in body fat ${ }^{48}$.

In the NEAT001 study, DRV/r plus RAL was not associated with a decline in renal function at week 96, but there were statistically significant increases in total cholesterol, LDL cholesterol, and HDL-cholesterol in RAL-based therapy?.

\section{Drug-Drug interaction}

The clinical pharmacology of dual therapy should be considered to identify appropriate combinations.

The $\mathrm{PI} / \mathrm{r}$ are CYP3A4 inhibitors and can affect the pharmacokinetics of different drugs.

Dual therapies with NRTIs and $\mathrm{PI} / \mathrm{r}$ have a low rate of virologic failure; a key factor is the absence of drug interaction between these classes.

Selected dosing was MVC $150 \mathrm{mg}$ once daily in MVC-containing dual therapy; in a post hoc reanalysis of the MOTIVATE trial, no concentration relationship was found between the once- and twice-daily $\mathrm{arms}^{49}$. In this registration trial, however, the use of DRV/r was not allowed. The pharmacokinetic profile of MVC $150 \mathrm{mg}$ once daily dosed with DRV/r 800/100 mg reported a possible MVC suboptimal exposure in such combination and suggested that MVC exposure was dependent on ritonavir exposure, which is reduced in the absence of TDF/FTC ${ }^{50}$. A lower MVC exposure was related to virologic failure in switch strategy ${ }^{17}$ and could be a possible explanation for drug failure in ARVnaive patients (MODERN study ${ }^{8}$ ). The MVC exposure, on the other hand, was shown to be adequate when associated with a higher ritonavir dosage, for example with LPV/r $r^{51}$.

Thus, a dose increase to MVC $300 \mathrm{mg}$ once daily seems reasonable when associated at least with DRV/r once daily and ATV/r.

However, not only drug-drug interactions should be pointed out when the pharmacological compatibility of a dual regimen is considered. Unexpected rates of virologic failure in patients with high baseline viral loads in the ACTG 5162 study $^{7}$ and lower pharmacological performance of the same subgroup of patients in the NEAT001 study ${ }^{8}$ suggested some pharmacodynamic issues could play a role. Even if the combination of DRV/r plus RAL shows an adequate profile of virologic potency, the short half lives of both compounds 
lead to limited tolerance of the whole regimen. The latter could become clinically significant in the presence of high viral load and suboptimal adherence ${ }^{52}$.

In a similar way, even in dual $\mathrm{Pl}$-sparing, pharmacological compatibility could play a role. In 26 patients treated with MVC plus RAL and a third drug, switching to RAL plus MVC showed a high rate of failure at week 24 , and $60 \%$ of patients had MVC $\mathrm{C}_{\text {trough }}$ lower than minimum effective concentration for experienced patients $(50 \mathrm{ng} / \mathrm{ml})^{19}$. This is contrary to previous data on the MVC plus RAL plus ETV combination, where no excess rate of virologic failure was observed in a similar clinical setting and in the presence of comparable MVC plasma exposure ${ }^{52}$. This finding suggests that the pharmacodynamics of triple regimens could not be fully applicable to dual regimens even within a switch strategy.

The pharmacological compatibility of dual regimens is a key issue in the selection of appropriate combinations, and should rely not only on pharmacokinetics (ruling out of clinically significant drug-drug interactions), but on pharmacodynamic features as well (genetic barrier and tolerance).

\section{Conclusions}

Dual antiretroviral therapy is an investigational strategic approach in HIV therapeutics that seeks to ameliorate toxicities and costs, and further improve the quality of life by reducing the drug burden. A large number of regimen combinations have been tested with different efficacy and safety results. Overall, they tend to be less effective than recommended triple regimens, with the risk of virologic failure being particularly greater in subjects with plasma HIV/RNA $\geq$ 100,000 copies/ml and CD4 $\leq 200 / m m c$. Accordingly, dual antiretroviral therapy should not be openly recommended and only be used with caution in ARV-naive patients. Dual therapies with lamivudine plus PIs seem to be efficacious initially, based on studies conducted either in ARV-naive subjects or as simplification strategies. More data and longer follow-up is needed for switch studies testing dual therapy including CCR5 antagonists and integrase inhibitors.

\section{References}

1. Panel on Antiretroviral Guidelines for Adults and Adolescents: Guidelines for the use of antiretroviral agents in HIV-1-infected adults and adolescents. Department of Health and Human Services. February 12, 2013. Available at: http://www.aidsinfo.nih.gov/ContentFiles/AdultandAdolescentGL.pdf

2. Reynes J, Trinh R, Pulido F, et al. Lopinavir/ritonavir combined with raltegravir or tenofovir/emtricitabine in antiretroviral-naive subjects: 96-weeks results of the PROGRESS study. AIDS Res Hum Retroviruses. 2013;29:256-65.

3. Nozza S, Galli L, Antinori A, et al. Maraviroc $150 \mathrm{mg}$ daily plus lopinavir/ ritonavir, a nucleoside/nucleotide reverse transcriptase inhibitor-sparing regimen for HIV-infected naive patients: 48-week final results of VEMAN study. Clin Microbiol Infect. 2015;21(5):510.e.1-9.

4. Mills A, Mildvan D, Podzamczer D, et al. Maraviroc once daily nucleoside analog-sparing regimen in treatment-naïve patients: randomised, openlabel pilot study. J Acquir Immune Defic Syndr. 2013;62:164-70.

5. Kozal MJ, Lupo S, DeJesus E, et al. A nucleoside- and ritonavir-sparing regimen containing atazanavir plus raltegravir in antiretroviral treatmentnaïve HIV-infected patients: SPARTAN study results. HIV Clin Trials. 2012;13:119-30.

6. Raffi F, Babiker AG, Richert L, et al. Ritonavir-boosted darunavir combined with raltegravir or tenofovir-emtricitabine in antiretroviral-naive adults infected with HIV-1: 96 week results from the NEAT001/ANRS143 randomised non-inferiority trial. Lancet. 2014;384:1942-51.

7. Taiwo B, Zheng L, Gallien S, et al. Efficacy of a nucleoside-sparing regimen of darunavir/ritonavir plus raltegravir in treatment-naive HIV1-infected patients (ACTG A5262). AIDS. 2011;25:2113-22.

8. Stellbrink HJ, Pulik P, Szlavik J, et al. Maraviroc (MVC) dosed once daily with darunavir/ritonavir (DRV/r) in a 2 drug-regimen compared to emtricitabine/tenofovir (TDF/FTC) with DRV/r; 48-week results from MODERN (Study A4001095). AIDS 2014. 20th International AIDS Conference. July, 2014. Melbourne. [Abstract TUAB0101]

9. Cahn P, Andrade-Villanueva J, Arribas JR, et al. Dual therapy with lopinavir and ritonavir plus lamivudine versus triple therapy with lopinavir and ritonavir plus two nucleoside reverse transcriptase inhibitors in antiretroviral-therapy-naive adults with HIV-1 infection: 48 week results of the randomised, open label, non-inferiority GARDEL trial. Lancet Infect Dis. 2014; 14:572-80.

10. Negredo E, Moltó J, Burger D, et al. Lopinavir/ritonavir plus nevirapine as a nucleoside-sparing approach in antiretroviral-experienced patients (NEKA study). J Acquir Immune Defic Syndr. 2005;38:47-52.

11. Negredo E, Miró O, Rodríguez-Santiago B, et al. Improvement of mitochondrial toxicity in patients receiving a nucleoside reverse-transcriptase inhibitor-sparing strategy: results from the Multicenter Study with Nevirapine and Kaletra (MULTINEKA). Clin Infect Dis. 2009:49:892-900

12. Tyrer M, Swaden L, Marshall NJ, Johnson M. Switching to dual therapy with darunavir/ritonavir and etravirine: a simplification strategy. $10^{\text {th }} \ln$ ternational Congress on Drug Therapy in HIV Infection. November 7-11, 2010. Glasgow. [Abstract P051].

13. Ofotokun I, Sheth AN, Sanford SE, et al. A switch in therapy to a reverse transcriptase inhibitor sparing combination of lopinavir/ritonavir and raltegravir in virologically suppressed HIV-infected patients: a pilot randomized trial to assess efficacy and safety profile: the KITE study. AIDS Res Hum Retroviruses. 2012;28:1196-206.

14. Di Giambenedetto S, Fabbiani M, Colafigli M, et al. Safety and feasibility of treatment simplification to atazanavir/ritonavir + lamivudine in HIVinfected patients on stable treatment with two nucleos(t)ide reverse transcriptase inhibitors + atazanavir/ritonavir with virological suppression (Atazanavir and Lamivudine for treatment Simplification, AtLaS pilot study). J Antimicrob Chemother. 2013;68:1364-72.

15. Pérez-Molina JA, Rivero A, Pasquau J, et al. Safety and Efficacy of Switching to Dual Therapy (Atazanavir/Ritonavir+Lamivudine) vs. Triple Therapy (Atazanavir/Ritonavir+Two Nucleos(t)ides) in Patients on Virologically Stable Antiretroviral Therapy: 24-week Interim Analysis from a Randomized Clinical Trial (SALT Study). 14th EACS, October 16-19 2013, Brussels, Belgium. [Abstract PE7/1].

16. Macías J, Recio E, Márquez M, et al. Efficacy and safety of once-daily Maraviroc plus Ritonavir-boosted Darunavir in pretreated HIV-infected patients in a real-life setting. HIV Medicine. 2014;15:417-24.

17. Obermaier M, Walter $\mathrm{H}$, Wienbreyer A, et al. Lower Maraviroc Plasma Levels in Combination with Darunavir than with Other Protease Inhibitors Was Associated to Virological Failure - 24 Week Analysis of the MITOX Study. 14 $4^{\text {th }}$ EACS, October 16-19 2013, Brussels, Belgium. [Abstract PE10/15].

18. Katlama C, Assoumou L, Valantin MA, et al. Maraviroc plus raltegravir failed to maintain virological suppression in HIV-infected patients with lipohypertrophy: results from the ROCnRAL ANRS 157 study. J Antimicrob Chemother. 2014;69:1648-52.

19. Nozza S, Bigoloni A, Calcagno A, et al. Viral rebound after switch to maraviroc/raltegravir dual therapy in highly experienced and virologically suppressed patients with HIV-1 infection. J Antimicrob Chemother. 2014;69:1436-9.

20. Cotte L, Durant J, Brochier C, et al. Safety and efficacy of a MaravirocRaltegravir combination following a 6 month induction phase with Maraviroc-Raltegravir-Tenofovir-Emtricitabine in naïve HIV-1 infected patients with CCR5 Virus: interim analysis of the No Nuc No Boost study IAS 2013; Kuala Lumpur, Malaysia. [Abstract WEPE511].

21. Calin R, Paris L, Simon A, et al. Dual etravirine/raltegravir combination in virologically suppressed HIV-1-infected patients on antiretroviral therapy. Antivir Ther. 2012;17:1601-4. 
22. Monteiro P, Perez I, Laguno M, et al. Dual therapy with etravirine plus raltegravir for virologically suppressed HIV-infected patients: a pilot study. J Antimicrob Chemother. 2014;69:742-8

23. D'Arminio Monforte A, Gianotti N, Cozzi-Lepri A, et al. Durability of Lopinavir/ritonavir mono-therapy in individuals with viral load $\leq 50$ copies/ $\mathrm{mL}$ in the observational setting. Antivir Ther. 2014;19:319-24.

24. Henrich TJ, Hu Z, Li JZ, et al. Long-term reduction in peripheral blood HIV type 1 reservoirs following reduced-intensity conditioning allogeneic stem cell transplantation. J Infect Dis. 2013;207:1694-702.

25. Guiguet M, Ghosn J, Duvivier C, et al. Boosted protease inhibitor monotherapy as a maintenance strategy: an observational study. AIDS. 2012;26:2345-50

26. Sidonie LN, Flandre P, Valantin MA, et al. Factors associated with virological failure in HIV-1-infected patients receiving darunavir/ritonavir monotherapy. J Infect Dis. 2011;204:1211-6.

27. Maldarelli F, Palmer S, King MS, et al. ART suppresses plasma HIVRNA to a stable set point predicted by pretherapy viremia. PLoS Pathog. 2007:3:e46.

28. Prazuck T, Zucman D, Avettand-Fènoël V, et al. Long-term HIV-1 virologic control in patients on a dual NRTI regimen. HIV Clin Trials. 2013 14:120-6

29. Taiwo B, Matining RM, Zheng $L$, et al. Associations of $T$ cell activation and inflammatory biomarkers with virological response to darunavir/ ritonavir plus raltegravir therapy. J Antimicrob Chemother. 2013;68 1857-61.

30. Svicher $V$, Cento $V$, Rozera $G$, et al. The genotypic false positive rate determined by $V 3$ population sequencing can predict the burden of HIV-1 CXCR4-using species detected by pyrosequencing. PLoS One. 2013:8:e53603.

31. Antinori A, Marcotullio S, Ammassari A, et al. Italian guidelines fo the use of antiretroviral agents and the diagnostic-clinical management of HIV-1 infected persons. Update 2011. New Microbiol. 2012 35:113-59.

32. Piconi S, Trabattoni D, Gori A, et al. Immune activation, apoptosis, and Treg activity are associated with persistently reduced CD4+ T-cell counts during antiretroviral therapy. AIDS. 2010;24:1991-2000.

33. Gaardbo JC, Hartling HJ, Gerstoft J, Nielsen SD. Incomplete immune recovery in HIV infection: mechanisms, relevance for clinical care, and possible solutions. Clin Dev Immunol. 2012;2012:670957.

34. Pinzone MR, Di Rosa M, Cacopardo B, Nunnari G. HIV RNA suppression and immune restoration: can we do better? Clin Dev Immunol. 2012 2012:515962.

35. D'Ettorre G, Paiardini M, Ceccarelli G, Silvestri G, Vullo V. HIV-associated immune activation: from bench to bedside. AIDS Res Hum Retroviruses. 2011:27:355-64.

36. Sandler NG, Wand $H$, Roque A, et al. Plasma levels of soluble CD14 independently predict mortality in HIV infection. J Infect Dis. 2011;203: 780-90.

37. Marchetti G, Cozzi-Lepri A, Merlini E, et al. Microbial translocation predicts disease progression of HIV-infected antiretroviral-naive patients with high CD4+ cell count. AIDS. 2011;25:1385-94.
38. Taaffe JE, Bosinger SE, Del Prete GQ et al CCR5 blockade is well tolerated and induces changes in the tissue distribution of CCR5+ and CD25+ T cells in healthy, SIV-uninfected rhesus macaques. J Med Primatol. 2012:41:24-42.

39. Hunt PW, Lederman MM, Deeks SG. Response: Maraviroc intensification and microbial translocation. Blood. 2013;122:2283-4

40. Wilkin TJ, Lalama CM, McKinnon J, et al. A pilot trial of adding maraviroc to suppressive antiretroviral therapy for suboptimal CD4+ T-cell recovery despite sustained virologic suppression: ACTG A5256. J Infect Dis. 2012;206:534-42.

41. Pallikkuth S. Fischl MA, Pahwa S. Combination antiretroviral therapy with raltegravir leads to rapid immunologic reconstitution in treatment-naive patients with chronic HIV infection. J Infect Dis. 2013;208:1613-23.

42. Rusconi S, Vitiello P, Adorni F, et al. Maraviroc as intensification strategy in HIV-1 positive patients with deficient immunological response: an Italian randomized clinical trial. PLoS One. 2013;8:e80157.

43. Montessori V, Press N, Harris M, Akagi L, Montaner JS. Adverse effects of antiretroviral therapy for HIV infection. CMAJ. 2004;170:229-38.

44. Burgos J, Crespo M, Falcó V, et al. Dual therapy based on a ritonavirboosted protease inhibitor as a novel salvage strategy for HIV-1-infected patients on a failing antiretroviral regimen. J Antimicrob Chemother. 2012:67:2479-86

45. Allavena C, Ferré V, Brunet-François C, et al. Efficacy and tolerability of a nucleoside reverse transcriptase inhibitor-sparing combination of lopinavir/ritonavir and efavirenz in HIV-1 infected patients. J Acquir Immune Defic Syndr. 2005;39:300-6.

46. Di Biagio A, Ricci E, Viscoli C, et al. The use of nucleoside reverse transcriptase inhibitors sparing regimens in treatment-experienced HIV1 infected patients. Curr HIV Res. 2013;1:179-86.

47. Tebas P, Zhang J, Yarasheski K, et al. Switching to a protease inhibitorcontaining, nucleoside-sparing regimen (lopinavir/ritonavir plus efavirenz) increases limb fat but raises serum lipid levels: results of a prospective randomized trial (AIDS clinical trial group 5125s). J Acquir Immune Defic Syndr. 2007;45:193-200.

48. Jacqmin P, Wade JR, Weatherley B, Snoeck E, Marshall S, McFadyen $\mathrm{L}$. Assessment of maraviroc exposure-response relationship at 48 weeks in treatment-experienced HIV-1-infected patients in the MOTIVATE studies. CPT Pharmacometrics Syst Pharmacol. 2013;2:e64.

49. Mora Peris B, Croucher A, Else LJ, et al. Pharmacokinetic profile and safety of $150 \mathrm{mg}$ of maraviroc dosed with $800 / 100 \mathrm{mg}$ of darunavir/ ritonavir all once daily, with and without nucleoside analogues, in HIVinfected subjects. J Antimicrob Chemother. 2013:68:1348-53.

50. Calcagno A, Nozza S, Gonzalez de Requena D, et al. Pharmacokinetics of maraviroc administered $150 \mathrm{mg}$ once daily in association with lopinavir/ritonavir in HIV-positive treatment-naïve patients, J Antimicrob Chemother. 2013:68:1686-8.

51. Sandkovsky U, Swindells S, Robbins BL, Nelson SR, Acosta EP, Fletcher CV. Measurement of plasma and intracellular concentrations of raltegravir in patients with HIV infection. AIDS. 2012:26:2257-9.

52. Calcagno A, Nozza S, Bonora S, et al. Pharmacokinetics of the raltegravir/ maraviroc/etravirine combination. J Antimicrob Chemother. 2011;66:1932-4. 